\title{
In Vitro Photothermal Destruction of Cancer Cells Using Gold Nanorods and Pulsed-Train Near-Infrared Laser
}

\author{
Jui-Teng Lin, ${ }^{1}$ Yueh-Sheng Chiang, ${ }^{2}$ Guang-Hong Lin, ${ }^{3}$ Hsinyu Lee, ${ }^{3}$ and Hsia-Wei Liu ${ }^{4}$ \\ ${ }^{1}$ New Vision Inc., 268-1 (11F), Han Sheng E. Road, Banciao, New Taipei City 22066, Taiwan \\ ${ }^{2}$ The Graduate Institute of Applied Science and Engineering, Fun Jen Catholic University, 510 Chung Cheng Road, \\ Hsinchuang, New Taipei City 24205, Taiwan \\ ${ }^{3}$ Department of Life Science, National Taiwan University, No. 1, Sec. 4, Roosevelt Road, Taipei 10617, Taiwan \\ ${ }^{4}$ Department of Life Science, College of Science and Engineering, Fun Jen Catholic University, 510 Chung Cheng Road, \\ Hsinchuang, New Taipei City 24205, Taiwan \\ Correspondence should be addressed to Hsia-Wei Liu,079336@mail.fju.edu.tw
}

Received 27 March 2012; Accepted 25 June 2012

Academic Editor: Yanbao Zhao

Copyright (๑) 2012 Jui-Teng Lin et al. This is an open access article distributed under the Creative Commons Attribution License, which permits unrestricted use, distribution, and reproduction in any medium, provided the original work is properly cited.

\begin{abstract}
We present a novel pulsed-train near-IR diode laser system with real-time temperature monitoring of the laser-heated cancer cell mixed in gold nanorod solution. Near-IR diode laser at $808 \mathrm{~nm}$ matching the gold nanorod absorption peak (with an aspect ratio about 4.0) was used in this study. Both surface and volume temperatures were measured and kept above $43^{\circ} \mathrm{C}$, the temperature for cancer cells destruction. The irradiation time needed in our pulsed-train system with higher laser fluence for killing the cancel cells is about 1-3 minutes, much shorter than conventional methods (5-10 minutes). Cell viabilities in gold nanorod mixed and controlled solutions are studied by green fluorescence.
\end{abstract}

\section{Introduction}

The use of nanoparticles in medicine is one of the important applications of nanotechnology. These include bioimaging and biosensing, drug delivery, cancer cell diagnostics, and therapeutics [1-10]. By changing the shape of nanoparticles from spheres to nanorods, the absorption and scattering wavelengths change from visible to the near infrared (NIR) region and therefore offer the advantages of larger optical cross-section and much deeper penetration depth in tissues $[8,9,11,12]$. Recent studies have shown that gold nanorods (GNRs) conjugated to antibodies [2] could be used for selective and efficient photothermal therapy. More recently, Lin et al. [11] proposed the use of a diode laser system having multiple wavelengths for more efficient treatment of cancer tumor.

Although NIR lasers for cancer therapy offers much deeper tissue penetration depths than that of visible lasers, they are limited to the normal tissues. In cancer tissues having GNRs components, its absorptions at the resonant wavelength increase significantly to about 1.5 to $5.0 \mathrm{~cm}^{-1}$, depending on the GNRs concentrations. Therefore the penetration depth in cancer tissue or tumor is still limited to about 0.1 to $0.3 \mathrm{~cm}$. Overheating on the surface area of targeted tissues is always an issue to be overcome. In addition, the distribution of the GNRs aspect ratios and their concentrations inside the cancer tissues or tumors are also difficult to be controlled for perfectly matching the laser peak absorption. To overcome these intrinsic difficulties, this paper introduces the novel techniques to improve the efficiency and selectivity of cancer therapy using NIR lasers [11].

In this study, we will first introduce the concept of pulsed train technique and the experimental setup using real-time monitoring devices. The measured surface temperature will be measured and compared with theory. The transient effect due to the direct absorbing of the thermal couple tip will be discussed. Finally, the cell viabilities in GNR mixed and controlled solutions are studied by green fluorescence at various laser powers. 


\section{Material and Method}

2.1. The Concept of Pulse Train. As shown in Figure 1, we compare the surface temperature of the GNR solution resulted from (a) a single-pulse laser and (b) pulsed-train which has a controlled laser on-off operation such that the surface temperature is kept at an average value of $T^{*}$. The benefit of this pulse train technique [11] includes the control of surface temperature at a given width to avoid overheating, and the increase of the volume temperature which is not available by conventional method. It should be noted that the ratio of on-off time needs to be tested experimentally for achieving a preset, constant surface temperature, although the theoretical prediction is possible under ideal situation.

2.2. Experiment. As shown in Figure 2, near infrared (NIR) diode laser system (custom-made by New Vision Inc., Taiwan, http://www.nvi-laser.com/) with three wavelengths at 785, 808, and $852 \mathrm{~nm}$ fiber-coupled, bundled, and coupled to one single external fiber connected to a hand piece. Using a focusing lens (focal length $20 \mathrm{~mm}$ ), we obtain a collimated output beam having a spot size of $8.0 \mathrm{~mm}$. For this spot size, the output laser power $P$ is converted to the laser fluence by $F=P /$ area $=2 P\left(\mathrm{~W} / \mathrm{cm}^{2}\right)$. The container for the solution of GNRs mixed in the distilled water has a diameter of $7.5 \mathrm{~mm}$ comparable to the laser spot size to make sure that the whole area is illuminated uniformly. GNRs with the aspect ratio of 4.0 , corresponding to an absorption peak around $810 \mathrm{~nm}$, was used in the experiment. To estimate the extinction coefficient of the solution, we calibrate the laser transmission at a given wavelength by measuring the transmitted laser power $P$ and $P^{\prime}$ when passing through the container in the distilled water and in the GNRs-mixed solution, respectively. With the given $P$ and $P^{\prime}$, the value of $A$ is calculated by, $A=\ln \left(P / P^{\prime}\right)$, where $y$ is the height of the solutions. Various values of $A$ are available either by dilution of the distilled water or by using different laser wavelengths which are off the peak absorption.

Two T-type thermal couples (Omega Engineering Inc., USA) were inserted into the GNRs solution at $z=1.5$ and $5.0 \mathrm{~mm}$ to measure the temperature near the surface and inside the GNRs solution, respectively. Temperature profiles are real-time monitored and recorded by DAQ device (National Instrument, USA). LabVIEW software was used to design a feedback system in controlling the laser on/off times for a preset surface temperature. The on/off pulsed-train technique allows the surface temperature of the GNRs solution to remain at a preset value without overheating, whereas the volume temperature may be significantly increased comparing to that of NIR laser operated in a cw mode [11].

The cancer cells, SH-SY5Y, were derived from the American Type Culture Collection (ATCC) and cultured in DMEM/F12 medium, supplemented with 10\% FBS under a humidified atmosphere of $95 \%$ air and $5 \% \mathrm{CO}_{2}$ at $37^{\circ} \mathrm{C}$. Cells were subcultured after trypsinization (in a $0.5 \%(\mathrm{v} / \mathrm{v})$ trypsin solution, supplemented with $0.2 \%$ (v/v) EDTA).

We note that multiple pulsed laser has been used in thermal therapy, but in conventional method (CM) which

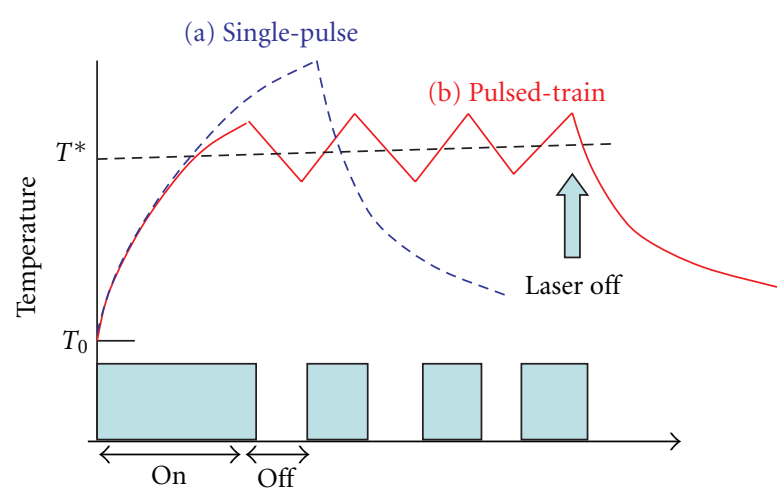

FIGURE 1: Schematic of the surface temperature of the GNR solution resulted from (a) a single-pulse laser, and (b) pulsed-train which has a controlled on-off operation such that the surface temperature is kept at an average value of $T^{*}[11]$.

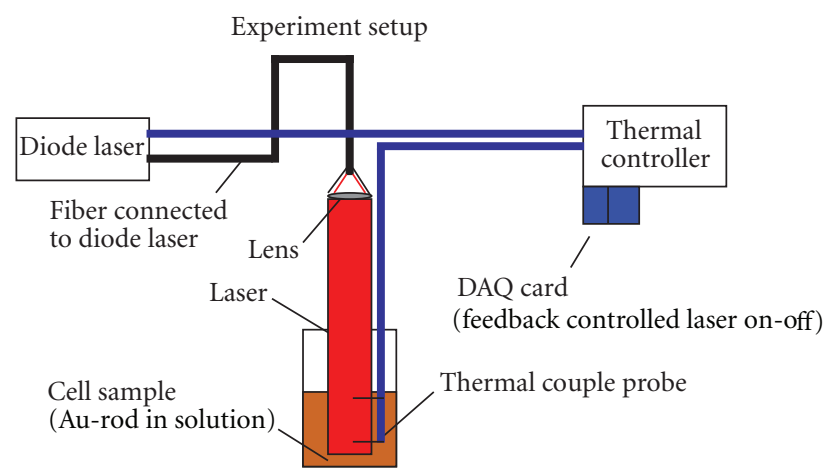

FIGURE 2: Experimental setup for a diode laser system with fibercoupled three wavelengths, bundled to one single external fiber connected to a hand piece and a collimation lens. Two thermal couples were inserted into the GNR solution at $z=1.5$ and $5.0 \mathrm{~mm}$ to measure the surface and volume temperature of the GNR solution [11].

does not have the preheating period as shown in Figure 2. The CM could not provide a preset surface temperature within a bandwidth (to avoid overheating). It is typically defined by the laser repetition rate (or number of pulses per unit time), that is, the on and off periods are the same and there is no preheating period. In our novel pulsed-train technique, the preset surface temperature is controlled by the combined techniques of (a) preheating period, and (b) the on-off ratio which is automatically controlled by a LabVIEW feedback signal to keep the preset surface temperature profile within a bandwidth, typically about $0.5-1.0^{\circ} \mathrm{C}$.

\section{Results and Discussions}

3.1. Surface Temperature. We first study the role of laser fluence $(F)$. We measure the laser illumination time needed (defined as $t_{2}$, in seconds) for the near-surface $(z=1.5 \mathrm{~mm})$ temperature increase of $10^{\circ} \mathrm{C}$ (from an initial temperature $25^{\circ} \mathrm{C}$ to $35^{\circ} \mathrm{C}$ ). Figure 3 shows the measured $t_{2}$ for a diode laser at $808 \mathrm{~nm}$ (with measured $A=3.5 \mathrm{~cm}^{-1}$ ), compared 


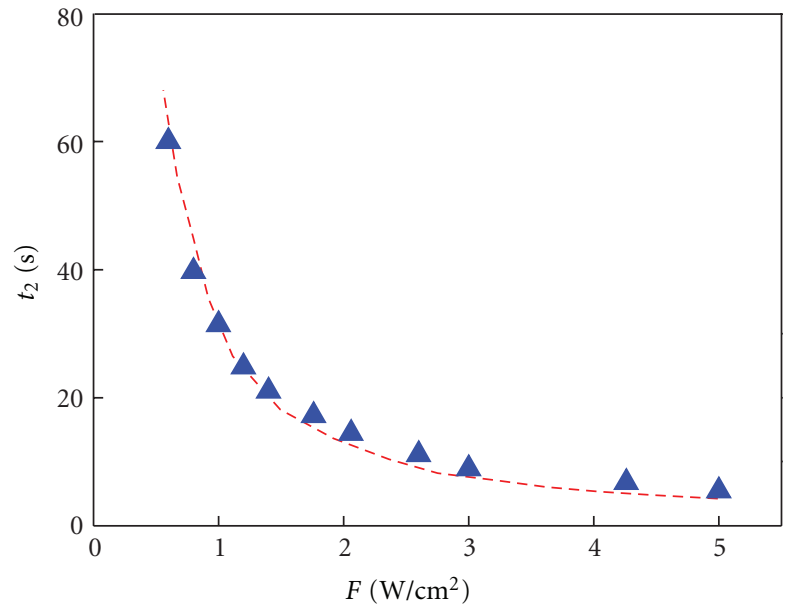

FIGURE 3: Laser illumination time needed (defined as $t_{2}$, in seconds) for the near-surface $(z=1.5 \mathrm{~mm})$ for surface temperature increase of $10^{\circ} \mathrm{C}$ as a function of the laser fluence, using diode laser at $808 \mathrm{~nm}$ (with measured $A=3.5 \mathrm{~cm}^{-1}$ ). Measured data are shown in triangles and compared with theoretical dashed curve.

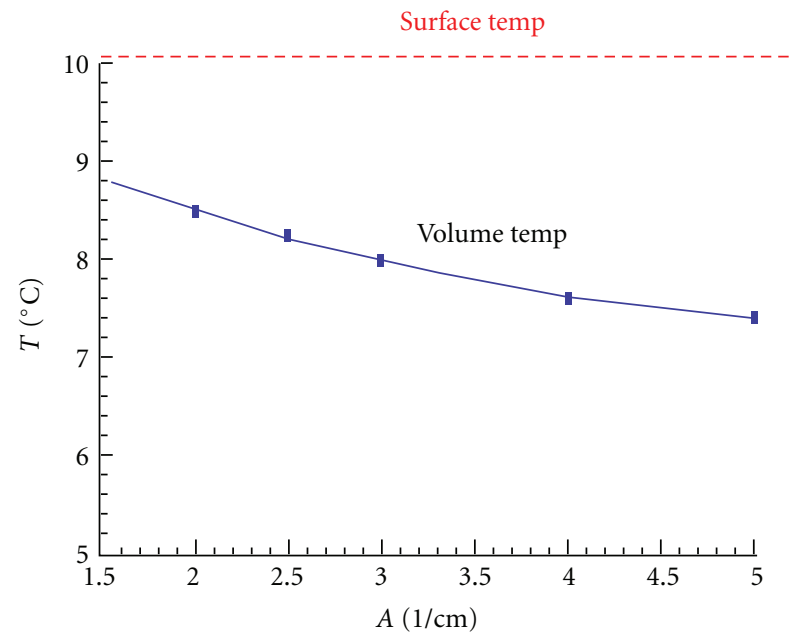

FIGURE 4: The calculated volume temperature (at $z=5.0 \mathrm{~mm}$ ) under pulsed-train operation with a preset surface temperature increase of $10^{\circ} \mathrm{C}$ for laser fluence of $F=2.0\left(\mathrm{~W} / \mathrm{cm}^{2}\right)$.

with theoretical dashed-curve from the solution of the heat diffusion equation [11]. Higher $F$ achieves a faster temperature increase, but in a nonlinear fashion as one may expect from the theory [11].

We now present the role of the absorption coefficient $(A)$. Figure 4 shows the calculated volume temperature (at $z=5.0 \mathrm{~mm}$ ) at steady-state under pulsed-train operation with a preset surface temperature increase of $10^{\circ} \mathrm{C}$ for laser fluence of $F=2.0\left(\mathrm{~W} / \mathrm{cm}^{2}\right)$. These results predict that smaller absorption coefficient will result in a higher volume temperature, for fixed laser fluence and under our on-off pulse train technique. The concept of optimal absorption coefficient $(A)$ may be realized by the laser source term in the heat diffusion equation [11], $A \exp [-A z]$, which has a maximum when $A=1 / z$.

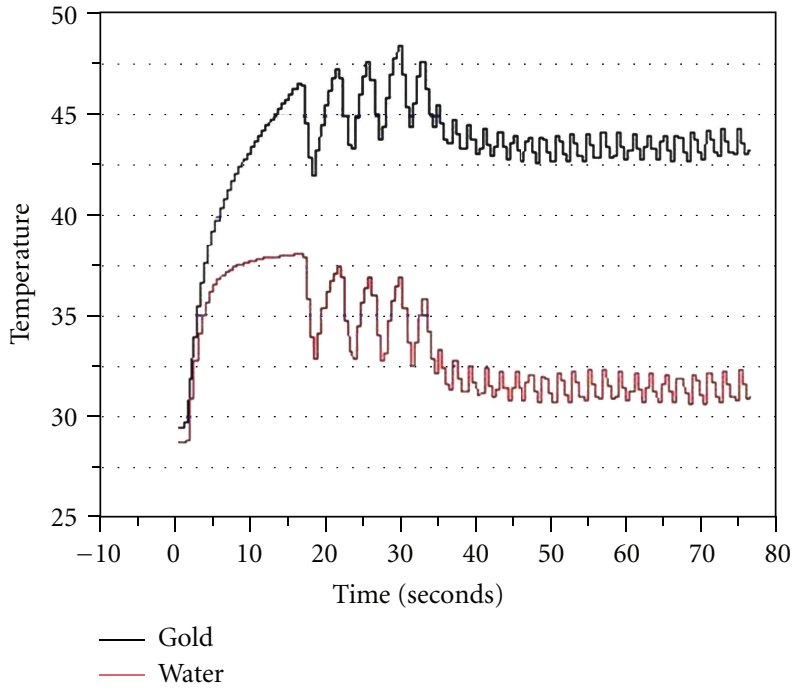

FIGURE 5: The transient temperature profiles attributed from the thermal couple only (measured in pure water, lower curve) and in the GNR solution (top curve).

It was also known that most of the cancer cells will be killed when its temperature reaches about $45^{\circ} \mathrm{C}$. Therefore, we preset our calculated temperature increase at $10^{\circ} \mathrm{C}$ (from an initial solution temperature about $35^{\circ} \mathrm{C}$ ) in GNR solution for cancer cells killing. The thermal parameters $(k, K)$ were chosen for typical skin tissue, and the $A$ values were measured from the GNR solution at a given concentration at a given laser wavelength in our simulation model. For real cancer tumor tissues, the situation could be much more complex. For example, the distribution of the GNR may not be uniform inside the tumor and the actual GNR concentration in the cancer tumor may not be available. In addition, multilayers of the skin covering the tumor tissues may affect the laser penetration depth and energy profile. Our simplified 1D simulation and the measurements based on GNR solution, therefore, may overestimate the overall laser efficiency in real tumor tissues. However, the general trends and the temperature profiles described in this paper should remain, in particular, the feature of the pulsed-train technique producing high volume temperature at a given optimal absorption condition.

3.2. Measurement Errors. In the experimental setup shown by Figure 2, the thermal couples placed within the GNR solution are in the path of the laser beam. Therefore, one should expect the thermal couple is absorbing some of the laser light, thus making the transient temperature attributed from both the thermal couples and the GNR solution. To evaluate the net temperature increase due to GNR $\left(d T_{1}\right)$, we have also measured the thermal couple temperature increase in pure water $\left(d T_{2}\right)$ as the controlled sample as shown in Figure 5. For a given laser power of $1.2 \mathrm{~W}$, and an initial temperature of $28^{\circ} \mathrm{C}$, the controlled water-solution showed a typical $d T_{2}$ about $6^{\circ} \mathrm{C}$ which is much lower than that of GNR solution, about $16^{\circ} \mathrm{C}$. The steady state temperature profiles 


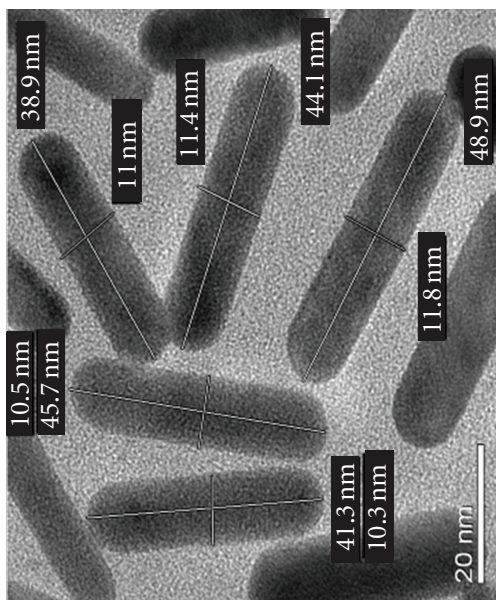

(a)

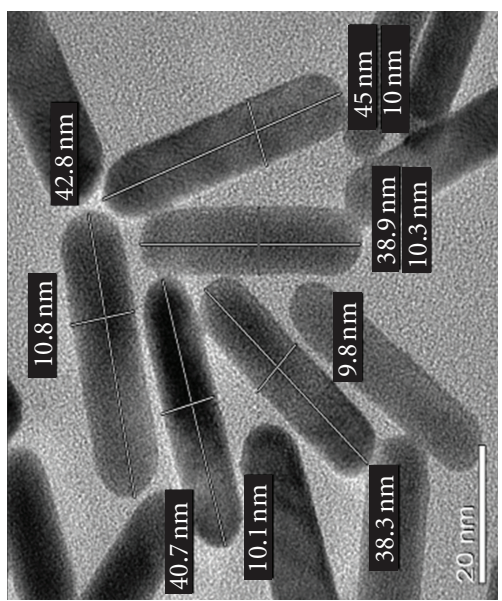

(b)

Figure 6: The distribution of gold nanorods in water solution shows length range of 38.3 to $45.7 \mathrm{~nm}$ (mean $42.5 \pm 3.5 \mathrm{~nm}$ ) and width of 9.8 to $11.0 \mathrm{~mm}$ (mean $10.6 \pm 0.6 \mathrm{~nm}$ ).

(defined as the profiles under the pulse-train mode) provide the evidence that the $10^{\circ} \mathrm{C}$ temperature difference of $d T_{1}$ and $d T_{2}$ is indeed due to the absorption of laser energy due to the GNR solution which is in thermal equivalent to the thermal couples. The initial transient temperature increase profile in GMR solution, however, is mainly attributed from the thermal couples which have much faster response to the laser heating than the GNR solution. To eliminate the effect caused by the direct thermal couple heating, one may insert the thermal couple outside the beam profile and measure the volume temperature via the thermal diffusion. In addition, an IR thermal camera may be used to monitor the realtime surface temperature profiles. However, the volume temperature cannot be measured by an IR camera. This study is mainly focusing on the volume temperature increase which is enhanced by the pulsed-train technique.

3.3. Nanorod Distribution. As shown in Figure 6, the gold nanorods in water solution (provided by Industrial Technology Research Institute, ITRI, Taiwan) have a length of 38.3 to $45.7 \mathrm{~nm}$ (mean $42.5 \pm 3.5 \mathrm{~nm}$ ) and width of 9.8 to $11.0 \mathrm{~mm}$ (mean $10.6 \pm 0.6 \mathrm{~nm}$ ). Therefore, the mean aspect ratio is $4.0 \pm 0.3$ corresponding to an absorption peak of $810 \pm 2 \mathrm{~nm}$. Figure 7 shows the absorption spectrum having a peak at $810 \mathrm{~nm}$.

3.4. Cell Viabilities. Cell viability after laser application was determined by using the live/dead viability/cytotoxicity kit (L-3224; Molecular Probes, Eugene, Ore). After being washed 3 times with PBS, the cell- GNRs were stained with a working reagent composed of $4 \mu \mathrm{L}$ of $2-\mathrm{mM}$ ethidium homodimer-1, $2 \mathrm{~mL}$ of PBS, and $1 \mu \mathrm{L}$ of 4 -mM calcein acetoxymethyl. The samples were incubated for $45 \mathrm{~min}$ at $37^{\circ} \mathrm{C}$ and identified under a confocal laser scanning microscope (TCS SP2; Leica Microsystems, Wetzlar, Germany).

Few damaged cells were observed when cells were treated with gold nanorods. Significant destruction of cells was

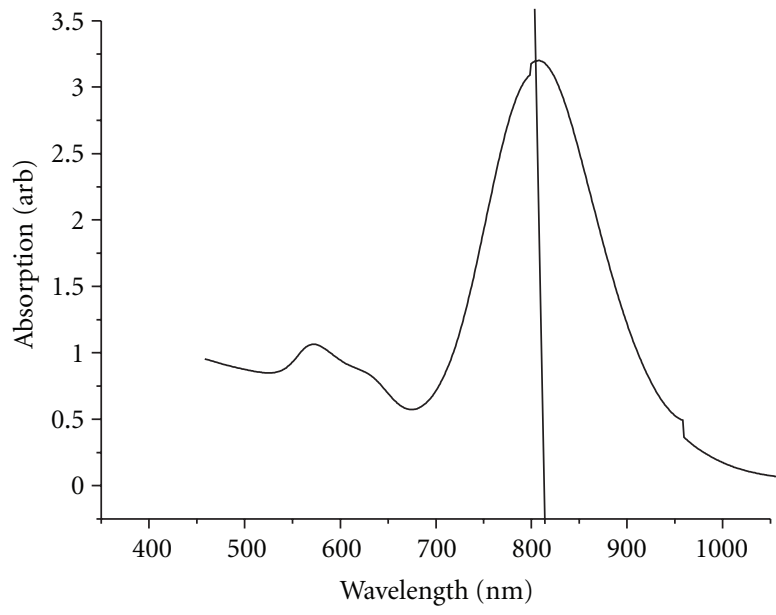

FIGURE 7: The absorption curve of gold nanorods in water solution with a mean aspect ratio 4.0 , showing a peak absorption at $810 \mathrm{~nm}$.

observed when the cells were exposed to laser irradiation at $1.0 \mathrm{~W}$ for 120 seconds in the presence of gold nanorods (Figure 8). These in vitro results indicate that cancer cells can be destroyed when mixed with gold nanorods and irradiated by near infrared laser.

Cell viabilities in GNR mixed and controlled solutions are studied by green fluorescence. As shown in Figure 9(a) for a given fixed laser power of $1.0 \mathrm{~W}$, longer laser irradiation time achieves lower cell viability (57\%). We expect a complete killing will need approximate 1-2 minutes depending on laser powers. Figure 9(b) also shows the viability at a given irradiation time of 60 seconds and at $1.35 \mathrm{~W}$ laser power, but for different preset surface temperatures $\left(T=43\right.$ and $\left.45^{\circ} \mathrm{C}\right)$. The higher preset surface temperature kills more cancer cells as expected. 


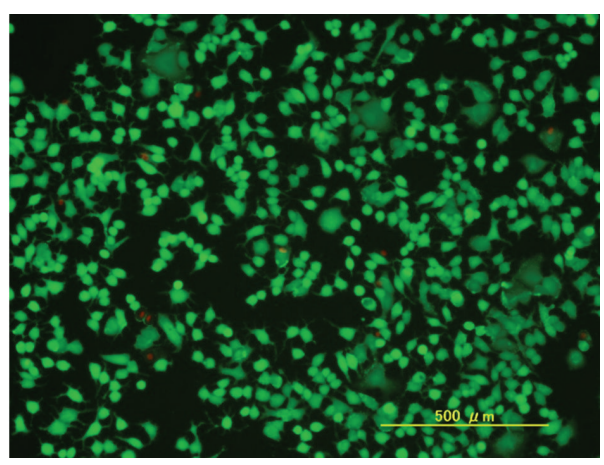

(a)

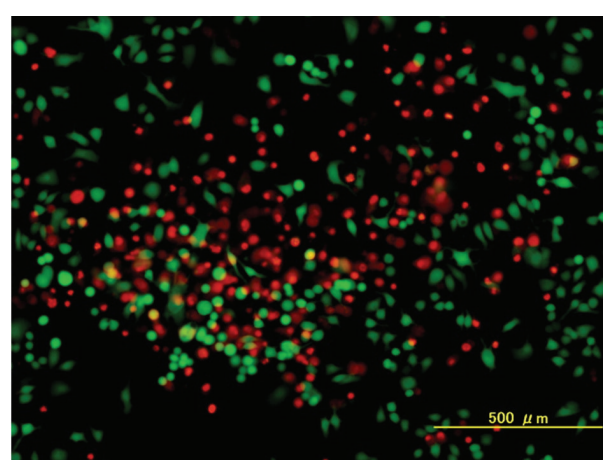

(b)

Figure 8: Cell viability of control cells (a) and after hyperthermia treatments (b). Cancer cells were stained with a live/dead cell viability assay to visualize live (green) and dead (red) cells, respectively.

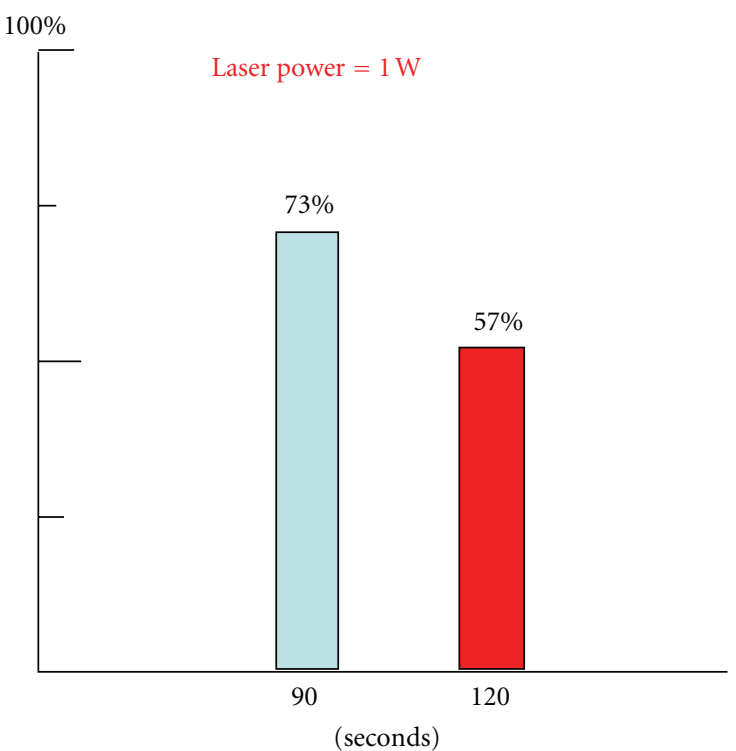

(a)

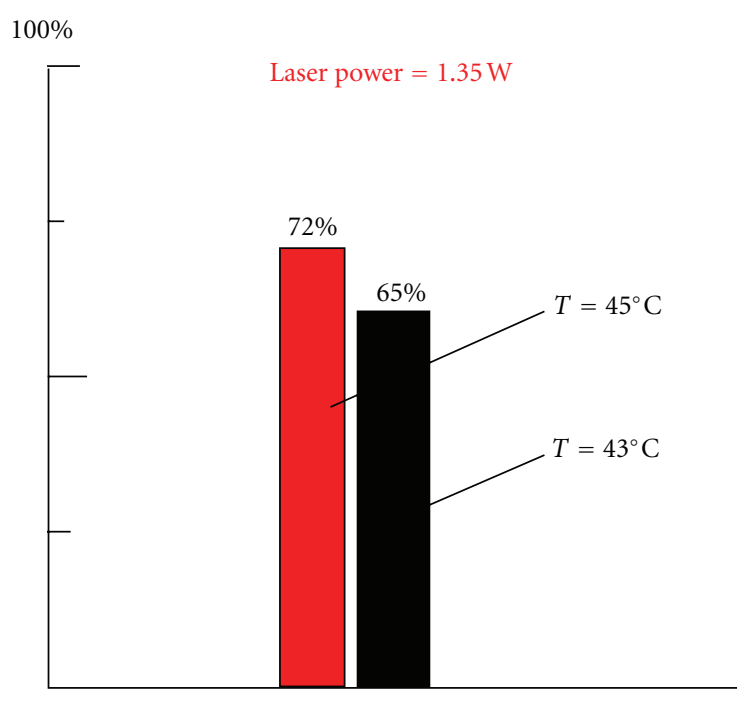

(b)

FIGURE 9: The cell viability at irradiation time of 90 and 120 seconds at laser power $1.0 \mathrm{~W}$ (a); (b) shows cell viability at a given irradiation time of 60 seconds and laser power of $1.35 \mathrm{~W}$, for different preset surface temperatures $\left(T=43\right.$ and $\left.45^{\circ} \mathrm{C}\right)$. The higher surface temperature kills more cancer cells as expected.

\section{Conclusion}

To conclude, the optimal laser operation for the surface and volume heating was achieved by a novel pulsed-train technique using an auto-controlled laser on/off time to meet the desired temperature for killing cancer cells. For a fixed laser fluency, GNR solution with smaller extinction coefficients $(A)$ provides higher volume temperature, but slower surface raising speed. The novel measured features are predicted by our theory. The pulsed-train technique when operated at optimal laser parameters provides a great potential for clinically high efficient laser therapy of large size cancer tumors. In vitro study of thermal destruction of cancer cells in laser-heated-GNR solution shows that the cell killing rate is proportional to the laser power and irradiation time.

\section{Acknowledgment}

This work is partially supported by the National Science Council (NSC) of Taiwan, Grant no. NSC 100-2221-E-030001.

\section{References}

[1] J. S. Murday, R. W. Siegel, J. Stein, and J. F. Wright, "Translational nanomedicine: status assessment and opportunities," Nanomedicine, vol. 5, no. 3, pp. 251-273, 2009.

[2] X. Huang, I. H. El-Sayed, W. Qian, and M. A. El-Sayed, "Cancer cells assemble and align gold nanorods conjugated to antibodies to produce highly enhanced, sharp, and polarized surface Raman spectra: a potential cancer diagnostic marker," Nano Letters, vol. 7, no. 6, pp. 1591-1597, 2007. 
[3] P. C. Chen, S. C. Mwakwari, and A. K. Oyelere, "Gold nanoparticles: from nanomedicine to nanosensing," Nanotechnology, Science and Applications, vol. 1, pp. 45-66, 2008.

[4] L. Tong, Q. Wei, A. Wei, and J. X. Cheng, "Gold nanorods as contrast agents for biological imaging: optical properties, surface conjugation and photothermal effects," Photochemistry and Photobiology, vol. 85, no. 1, pp. 21-32, 2009.

[5] J. L. West and N. J. Halas, "Engineered nanomaterials for biophotonics applications: improving sensing, imaging, and therapeutics," Annual Review of Biomedical Engineering, vol. 5, pp. 285-292, 2003.

[6] G. F. Paciotti, L. Myer, D. Weinreich et al., "Colloidal gold: a novel nanoparticle vector for tumor directed drug delivery," Drug Delivery, vol. 11, no. 3, pp. 169-183, 2004.

[7] L. R. Hirsch, R. J. Stafford, J. A. Bankson et al., "Nanoshellmediated near-infrared thermal therapy of tumors under magnetic resonance guidance," Proceedings of the National Academy of Sciences of the United States of America, vol. 100, no. 23, pp. 13549-13554, 2003.

[8] X. Huang, I. H. El-Sayed, W. Qian, and M. A. El-Sayed, "Cancer cell imaging and photothermal therapy in the nearinfrared region by using gold nanorods," Journal of the American Chemical Society, vol. 128, no. 6, pp. 2115-2120, 2006.

[9] I. H. El-Sayed, X. Huang, and M. A. El-Sayed, "Selective laser photo-thermal therapy of epithelial carcinoma using anti-EGFR antibody conjugated gold nanoparticles," Cancer Letters, vol. 239, no. 1, pp. 129-135, 2006.

[10] M. Everts, V. Saini, J. L. Leddon et al., "Covalently linked Au nanoparticles to a viral vector: potential for combined photothermal and gene cancer therapy," Nano Letters, vol. 6, no. 4, pp. 587-591, 2006.

[11] J. T. Lin, Y. L. Hong, and C. L. Chang, "Selective cancer therapy via IR-laser-excited gold nanorods," in Proceedings of the Optical Interactions with Tissues and Cells XXI, vol. 7562 of Proceedings of SPIE, p. 75620R-1, San Francisco, Calif, USA, January 2010.

[12] J. T. Lin, "Nonlinear optical theory and figure of merit of surface plasmon resonance of gold nanorods," Journal of Nanophotonoics, vol. 5, no. 1, Article ID 051506, 2011. 

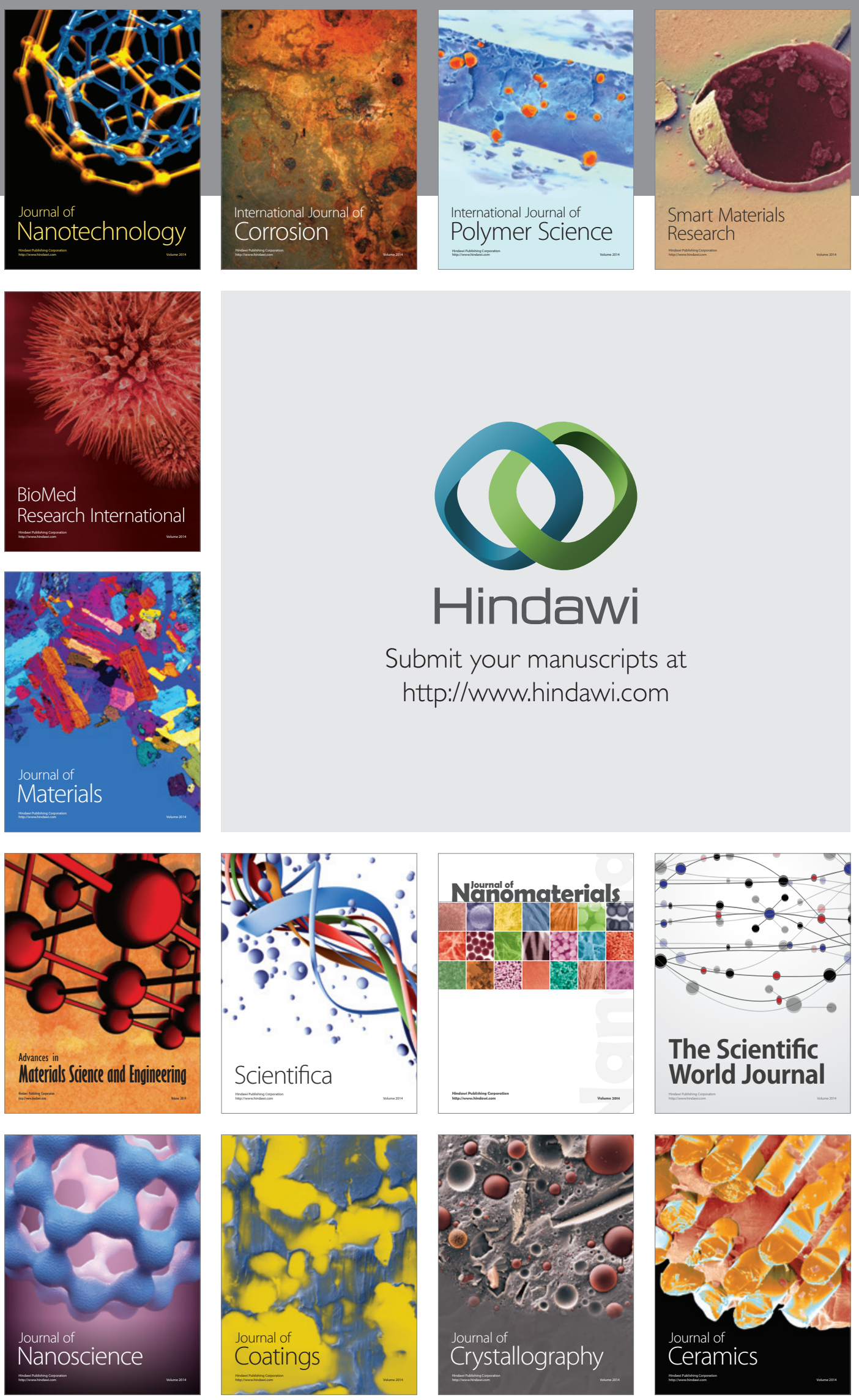

The Scientific World Journal

Submit your manuscripts at

http://www.hindawi.com

\section{World Journal}

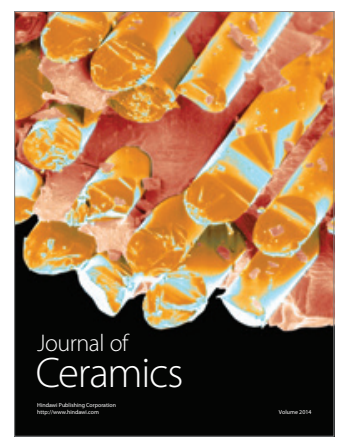

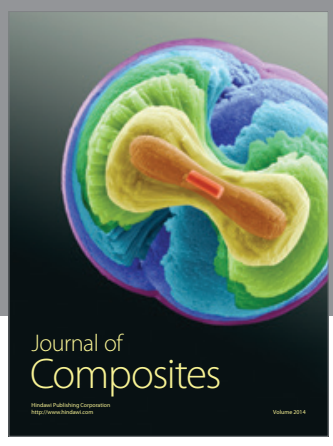
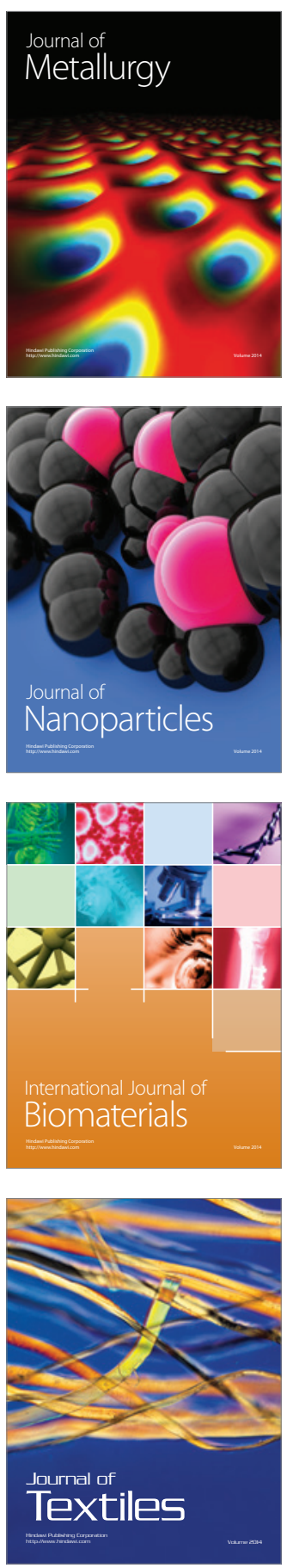